\title{
Existence of Solutions of a Partial Integrodifferential Equation with Thermostat and Time Delay
}

\author{
Carlo Bianca, ${ }^{1,2}$ Luca Guerrini, ${ }^{3}$ and Annie Lemarchand ${ }^{1,2}$ \\ ${ }^{1}$ Laboratoire de Physique Théorique de la Matière Condensée, Sorbonne Universités, UPMC Univ Paris 06, UMR 7600, Place Jussieu, \\ Case Courrier 121, 75252 Paris Cedex 05, France \\ ${ }^{2}$ CNRS, UMR 7600 LPTMC, Paris, France \\ ${ }^{3}$ Department of Management, Polytechnic University of Marche, 60121 Ancona, Italy
}

Correspondence should be addressed to Carlo Bianca; bianca@lptmc.jussieu.fr

Received 1 July 2014; Accepted 11 August 2014; Published 14 October 2014

Academic Editor: Xiao-Jun Yang

Copyright (C) 2014 Carlo Bianca et al. This is an open access article distributed under the Creative Commons Attribution License, which permits unrestricted use, distribution, and reproduction in any medium, provided the original work is properly cited.

This paper deals with the mathematical analysis of a retarded partial integrodifferential equation that belongs to the class of thermostatted kinetic equations with time delay. Specifically, the paper is devoted to the proof of the existence and uniqueness of mild solutions of the related Cauchy problem. The main result is obtained by employing integration along the characteristic curves and successive approximations sequence arguments. Applications and perspective are also discussed within the paper.

\section{Introduction}

The derivation and analysis of mathematical frameworks have recently gained much attention in the applied sciences and specifically for the modeling of complex phenomena occurring in biology, chemistry, vehicular traffic, crowd/swarm dynamics, economics, and social systems. At the origin of complex dynamics, there are interactions that occur in nonlinear fashion and randomly among the elements (particles, cells, and pedestrians) composing the complex system [1]. Complex systems are also characterized by emergent properties, which are properties of the system as a whole which do not exist at the individual element level.

Different ordinary differential equations and partial differential equations have been derived with the aim of obtaining an accurate description of complex phenomena. Moreover, thermostatted kinetic frameworks have been developed for the mathematical modeling of complex systems in physics and life sciences [2-5]. The goal of the thermostatted kinetic models is the possibility of modeling the interactions among the particles at the microscopic scale. In particular, these frameworks allow modeling the ability of the particles to express strategies.
The present paper deals with further developments of the thermostatted kinetic theory proposed in [6]. Specifically, this paper is devoted to the mathematical analysis of a partial integrodifferential equation with thermostat and time delay that belongs to the class of the thermostatted kinetic theory frameworks. The paper focuses on the proof of the existence and uniqueness of mild solutions of the related Cauchy problem. The main result is obtained by employing integration along the characteristic curves and successive approximations sequence arguments. Applications and perspective are also discussed within the paper.

The time delay is introduced in order to take into account the fact that most of the emerging behaviours occurring in complex systems at a certain time are strictly related to the interactions among the particles of the system at a previous time. In the pertinent literature, mathematical models with time delays have been proposed only in ODE-based models; see, among others, [7-16]. The introduction of the time delay has provoked the onset of fluctuations and Hopf bifurcation; see $[17-20]$.

It is worth stressing that, to the best of our knowledge, this is the first time that time delay is introduced into a thermostatted partial integrodifferential equation (kinetic). 
The contents of the present paper are developed through four more sections, which follow this introduction. In detail, Section 2 deals with the retarded partial integrodifferential equation and the related Cauchy problem; Section 3 is concerned with some preliminary results that are needed for the proof of the main result that is outlined in Section 4. Finally, Section 5 is devoted to a critical analysis of the proposed mathematical equation including research perspective and applications.

\section{The Retarded Integrodifferential Equation}

This paper is devoted to the result about the existence and uniqueness of a solution of the following Cauchy problem:

$$
\begin{gathered}
\partial_{t} f(t, u)+F \partial_{u}\left(f(t, u)\left(1-u \int_{D_{u}} u f(t, u) d u\right)\right) \\
=\eta J\left[f, f_{\tau}\right](t, u) \\
f(0, u)=f_{0}(u)
\end{gathered}
$$

where $f=f(t, u):[0,+\infty) \times D_{u} \rightarrow \mathbb{R}^{+}$is the unknown function, $f_{\tau}=f(t-\tau, u):[-\tau,+\infty) \times D_{u} \rightarrow \mathbb{R}^{+}, F, \eta \in \mathbb{R}^{+}$, and $J\left[f, f_{\tau}\right]=J\left[f, f_{\tau}\right](t, u)$ is the following integral operator:

$$
\begin{aligned}
J\left[f, f_{\tau}\right]= & \int_{D_{u} \times D_{u}} \mathscr{A}\left(u_{*}, u^{*}, u\right) f\left(t, u_{*}\right) \\
& \times f\left(t-\tau, u^{*}\right) d u_{*} d u^{*} \\
& -f(t, u) \int_{D_{u}} f\left(t-\tau, u^{*}\right) d u^{*}
\end{aligned}
$$

with $\mathscr{A}\left(u_{*}, u^{*}, u\right): D_{u} \times D_{u} \times D_{u} \rightarrow \mathbb{R}^{+}, \tau$ the time delay, and $f_{0}$ the initial datum.

In the partial integrodifferential equation with time delay

$$
\begin{aligned}
\partial_{t} f(t, u) & +F \partial_{u}\left(f(t, u)\left(1-u \int_{D_{u}} u f(t, u) d u\right)\right) \\
& =\eta J\left[f, f_{\tau}\right](t, u),
\end{aligned}
$$

$J\left[f, f_{\tau}\right](t, u)=G\left[f, f_{\tau}\right](t, u)-L\left[f, f_{\tau}\right](t, u)$ is the conservative interaction operator, which is splitted into the gain (of particles into state $u$ ) operator $G\left[f, f_{\tau}\right]=G\left[f, f_{\tau}\right](t, u)$ and the loss (of particles into state $u$ ) operator $L\left[f, f_{\tau}\right]=$ $L\left[f, f_{\tau}\right](t, u)$ :

$$
\begin{gathered}
G\left[f, f_{\tau}\right] \\
=\int_{D_{u} \times D_{u}} \mathscr{A}\left(u_{*}, u^{*}, u\right) f\left(t, u_{*}\right) f\left(t-\tau, u^{*}\right) d u_{*} d u^{*} \\
L\left[f, f_{\tau}\right]=f(t, u) \int_{D_{u}} f\left(t-\tau, u^{*}\right) d u^{*}
\end{gathered}
$$

Bearing all of the above in mind, and under suitable integrability assumptions on $f$, the pth-order moment of $f$ is defined as follows:

$$
\mathbb{E}_{p}[f](t)=\int_{D_{u}} u^{p} f(t, u) d u, \quad p \in \mathbb{N} .
$$

In particular, the zero-order, first-order, and second-order moments represent the density (mass), mean activation (linear momentum), and activation energy (kinetic energy), respectively. The term

$$
\mathscr{T}_{F}[f]:=F \partial_{u}\left(f(t, u)\left(1-u \int_{D_{u}} u f(t, u) d u\right)\right)
$$

is a damping operator that allows the control of the activation energy. This term is based on the Gaussian isokinetic thermostat (the interested reader is referred, among others, to [2123]).

In what follows, we assume that $f(t, u)=0$ for $(t, u) \epsilon$ $[-\tau, 0] \times D_{u}$ (initial function).

\section{Preliminary Results}

This section is concerned with some preliminary results that are at the basis of the main result of the present paper.

Lemma 1. The gain operator (4) satisfies, for all functions $f$ and $g$, the following identity:

$$
G\left[f, f_{\tau}\right]-G\left[g, g_{\tau}\right]=G\left[f-g, f_{\tau}\right]+G\left[g, f_{\tau}-g_{\tau}\right] .
$$

Proof. It is obtained by straightforward calculations.

The main result is based on the following assumptions on the probability density function $\mathscr{A}$.

$\left(\mathrm{A}_{1}\right)$ The probability density function $\mathscr{A}$ satisfies, for all $u_{*}, u^{*} \in D_{u}$, the following identity:

$$
\int_{D_{u}} \mathscr{A}\left(u_{*}, u^{*}, u\right) d u=1
$$

which models the conservation of particles.

$\left(\mathrm{A}_{2}\right)$ The probability density function $\mathscr{A}$ is an even function with respect to $u$; then, in particular,

$$
\int_{D_{u}} u \mathscr{A}\left(u_{*}, u^{*}, u\right) d u=0 .
$$

$\left(\mathrm{A}_{3}\right)$ The probability density function $\mathscr{A}$ satisfies, for all $u_{*}, u^{*} \in D_{u}$, the following identity:

$$
\int_{D_{u}} u^{2} \mathscr{A}\left(u_{*}, u^{*}, u\right) d u=u_{*}^{2} .
$$

Lemma 2. If the function $\mathscr{A}$ satisfies assumptions (9), (10), and (11), then

$$
\begin{gathered}
\int_{D_{u}} G\left[f, f_{\tau}\right](t, u) d u \\
=\left(\int_{D_{u}} f(t, u) d u\right)\left(\int_{D_{u}} f(t-\tau, u) d u\right), \\
\quad \int_{D_{u}} u^{2 p+1} G\left[f, f_{\tau}\right](t, u) d u=0, \quad p \in \mathbb{N}, \\
\int_{D_{u}} u^{2} G\left[f, f_{\tau}\right](t, u) d u \\
=\left(\int_{D_{u}} u^{2} f(t, u) d u\right)\left(\int_{D_{u}} f(t-\tau, u) d u\right) .
\end{gathered}
$$


Proof. Condition (9) implies

$$
\begin{aligned}
& \int_{D_{u}} G\left[f, f_{\tau}\right](t, u) d u \\
&= \int_{D_{u}}\left(\int_{D_{u} \times D_{u}} \mathscr{A}\left(u_{*}, u^{*}, u\right) f\left(t, u_{*}\right)\right. \\
&\left.\times f\left(t-\tau, u^{*}\right) d u_{*} d u^{*}\right) d u \\
&=\int_{D_{u} \times D_{u}}\left(\int_{D_{u}} \mathscr{A}\left(u_{*}, u^{*}, u\right) d u\right) \\
& \times f\left(t, u_{*}\right) f\left(t-\tau, u^{*}\right) d u_{*} d u^{*} \\
&=\left(\int_{D_{u}} f\left(t, u_{*}\right) d u^{*}\right)\left(\int_{D_{u}} f\left(t-\tau, u^{*}\right) d u^{*}\right) .
\end{aligned}
$$

Bearing in mind condition (10), we have

$$
\begin{aligned}
& \int_{D_{u}} u^{2 p+1} G\left[f, f_{\tau}\right](t, u) d u \\
&=\int_{D_{u}} u^{2 p+1}\left(\int_{D_{u} \times D_{u}} \mathscr{A}\left(u_{*}, u^{*}, u\right) f\left(t, u_{*}\right)\right. \\
&\left.\times f\left(t-\tau, u^{*}\right) d u_{*} d u^{*}\right) d u \\
&=\int_{D_{u} \times D_{u}}\left(\int_{D_{u}} u^{2 p+1} \mathscr{A}\left(u_{*}, u^{*}, u\right) d u\right) \\
& \quad \times f\left(t, u_{*}\right) f\left(t-\tau, u^{*}\right) d u_{*} d u^{*}=0 .
\end{aligned}
$$

Finally, condition (11) implies

$$
\begin{aligned}
\int_{D_{u}} u^{2} G\left[f, f_{\tau}\right](t, u) d u & \\
= & \int_{D_{u}} u^{2}\left(\int_{D_{u} \times D_{u}} \mathscr{A}\left(u_{*}, u^{*}, u\right) f\left(t, u_{*}\right)\right. \\
& \left.\times f\left(t-\tau, u^{*}\right) d u_{*} d u^{*}\right) d u \\
= & \int_{D_{u} \times D_{u}}\left(\int_{D_{u}} u^{2} \mathscr{A}\left(u_{*}, u^{*}, u\right) d u\right) \\
= & \int_{D_{u} \times D_{u}} u_{*}^{2} f\left(t, u_{*}\right) f\left(t-\tau, u^{*}\right) d u_{*} d u^{*} \\
= & \left(\int_{D_{u}} u_{*}^{2} f\left(t, u_{*}\right) d u_{*}\right)\left(\int_{D_{u}} f\left(t-\tau, u^{*}\right) d u^{*}\right) .
\end{aligned}
$$

Therefore, the proof of the lemma is concluded.

Bearing the previous lemma in mind, the evolution equation for the 1st-order moment of $f$ is stated in the following results.
Theorem 3. Assume that assumptions (9), (10), and (11) hold. If there exists a nonnegative solution $f$ of the partial integrodifferential equation (3) such that $f(t, u)=0$ as $u \in$ $\partial D_{u}$, then the 1st-order moment $\mathbb{E}_{1}[f](t)$ is solution of the following delayed first-order nonlinear ordinary differential equation:

$$
\begin{aligned}
\frac{d}{d t} \mathbb{E}_{1}[f](t)= & F\left[\mathbb{E}_{0}[f](t)-\left(\mathbb{E}_{1}[f](t)\right)^{2}\right] \\
& -\eta \mathbb{E}_{1}[f](t) \mathbb{E}_{0}[f](t-\tau) .
\end{aligned}
$$

Proof. The integral operator $J\left[f, f_{\tau}\right]$ can be written as follows:

$$
\begin{aligned}
J\left[f, f_{\tau}\right](t, u)= & G\left[f, f_{\tau}\right](t, u) \\
& -f(t, u) \int_{D_{u}} f\left(t-\tau, u^{*}\right) d u^{*}
\end{aligned}
$$

Multiplying both sides of $J\left[f, f_{\tau}\right]$ by $u$, integrating over $D_{u}$, and considering (10), we have

$$
\begin{aligned}
\int_{D_{u}} u J & {\left[f, f_{\tau}\right](t, u) d u } \\
& =-\left(\int_{D_{u}} u f(t, u) d u\right)\left(\int_{D_{u}} f(t-\tau, u) d u\right) \\
& =-\mathbb{E}_{1}[f](t) \mathbb{E}_{0}[f](t-\tau) .
\end{aligned}
$$

Since

$$
\begin{gathered}
\int_{D_{u}} u \partial_{u}\left(f(t, u)\left(1-u \int_{D_{u}} u f(t, u) d u\right)\right) d u \\
=\left(\mathbb{E}_{1}[f](t)\right)^{2}-\mathbb{E}_{0}[f](t),
\end{gathered}
$$

then we have the proof.

Corollary 4. Assume that assumptions (9), (10), and (11) hold. If there exists a nonnegative solution $f$ of the Cauchy problem (1) such that

(i) $\mathbb{E}_{0}[f](t)=\mathbb{E}_{0}[f](t-\tau)=1$,

(ii) $f(t, u)=0$ as $u \in \partial D_{u}$,

then the 1st-order moment $\mathbb{E}_{1}[f](t)$ reads as follows:

$$
\begin{aligned}
\beta(t) & :=\mathbb{E}_{1}[f](t) \\
= & \frac{\mathbb{E}_{1}^{+}\left(\mathbb{E}_{1}^{-}-\mathbb{E}_{1}^{0}\right)-\mathbb{E}_{1}^{-}\left(\mathbb{E}_{1}^{+}-\mathbb{E}_{1}^{0}\right) e^{-\left(\sqrt{\eta^{2}+4 F^{2}} / F\right) t}}{\left(\mathbb{E}_{1}^{-}-\mathbb{E}_{1}^{0}\right)-\left(\mathbb{E}_{1}^{+}-\mathbb{E}_{1}^{0}\right) e^{-\left(\sqrt{\eta^{2}+4 F^{2}} / F\right) t}},
\end{aligned}
$$

where

$$
\begin{gathered}
\mathbb{E}_{1}^{ \pm}=\frac{-\eta \pm \sqrt{\eta^{2}+4 F^{2}}}{2 F}, \\
\mathbb{E}_{1}^{0}=\mathbb{E}_{1}[f](0)=\int_{D_{u}} u f_{0} d u .
\end{gathered}
$$


Proof. The proof is obtained by coupling the delayed differential equation (18) with the initial condition $\mathbb{E}_{1}[f](0)=\mathbb{E}_{1}^{0}$. If $\mathbb{E}_{1}^{0}=\mathbb{E}_{1}^{+}$or $\mathbb{E}_{1}^{0}=\mathbb{E}_{1}^{-}$, then we have $\mathbb{E}_{1}[f](t)=\mathbb{E}_{1}^{0}$ for all $t>0$. Otherwise, the unique solution is function (22).

Theorem 5. Let $p$ be an odd number and $t \geq 0$. Assume that assumptions (9), (10), and (11) hold. Then, the pthorder moment of $f$ satisfies the following delayed ordinary differential equation:

$$
\begin{aligned}
\frac{d}{d t} \mathbb{E}_{p}[f](t) & \\
= & -\mathbb{E}_{p}[f](t)\left[p F \mathbb{E}_{1}[f](t)+\eta \mathbb{E}_{0}[f](t-\tau)\right] \\
& +p F \mathbb{E}_{p-1}[f](t) .
\end{aligned}
$$

Proof. The proof follows by multiplying both sides of (3) by $u^{p}$, taking into account assumptions (9), (10), and (11), and performing integration by parts on the thermostat term.

According to Corollary 4, (3) can be rewritten as follows:

$$
\partial_{t} f+F(1-u \beta(t)) \partial_{u} f+(\eta-F \beta(t)) f=G\left[f, f_{\tau}\right](t, u),
$$

and, after integrating along the characteristics, (25) reads as follows:

$$
\frac{d}{d t} f_{U}+(\eta-F \beta(t)) f_{U}=G_{U}\left[f, f_{\tau}\right](t, u),
$$

where

$$
\begin{aligned}
f_{U}(t, u) & :=f(t, U(t, u)), \\
G_{U}\left[f, f_{\tau}\right](t, u) & :=G\left[f, f_{\tau}\right](t, U(t, u)),
\end{aligned}
$$

being

$$
\begin{gathered}
U(t, u)=\varphi_{t}(u)=u e^{-\lambda(t)}+F e^{-\lambda(t)} \int_{0}^{t} e^{\lambda(s)} d s, \\
\lambda(t)=F \int_{0}^{t} \beta(s) d s, \\
u=\varphi_{t}^{-1}(U)=U e^{\lambda(t)}-F \int_{0}^{t} e^{\lambda(s)} d s .
\end{gathered}
$$

Bearing all of the above in mind, the integral form of (26) is

$$
\begin{array}{r}
f_{U}(t, u)=e^{-\Lambda(t)} f_{U}(0, u) \\
+e^{-\Lambda(t)} \int_{0}^{t} e^{\Lambda(\alpha)} G_{U}\left[f, f_{\tau}\right](\alpha, u) d \alpha, \\
\forall t \in[0, T],
\end{array}
$$

where $\Lambda(t)=\int_{0}^{t}(\eta-F \beta(s)) d s=\eta t-\lambda(t)$.

\section{Existence of Mild Solutions}

Definition 6. A function $f$ is said to be a mild solution to the Cauchy problem (1) on the time interval $[0, T]$ if $f(t, \cdot) \in L^{1}\left(D_{u}, d u\right)$ and $f$ is solution to the following integral equation:

$$
f(t, u)=\Phi_{f_{0}}\left[f, f_{\tau}\right](t, u),
$$

where

$$
\begin{aligned}
& \Phi_{f_{0}}\left[f, f_{\tau}\right](t, u) \\
&=e^{-\Lambda(t)} f_{0}\left(\varphi_{t}^{-1}(u)\right) \\
&+e^{-\Lambda(t)} \int_{0}^{t} e^{\Lambda(\alpha)} G\left[f, f_{\tau}\right]\left(\alpha, \varphi_{\alpha} \circ \varphi_{t}^{-1}(u)\right) d \alpha .
\end{aligned}
$$

Lemma 7. Let $\left\{f^{(n)}(t, u)\right\}_{n}$ be the following successive approximations sequence:

$$
\begin{gathered}
f^{(1)}(t, u)=0, \\
f^{(n)}(t, u)=\Phi_{f_{0}}\left[f^{(n-1)}, f_{\tau}^{(n-1)}\right](t, u), \quad n>1,
\end{gathered}
$$

where $f_{0}$ is a nonnegative function such that $\mathbb{E}_{0}\left[f_{0}\right](t)=1$. Then, $\left\{f^{(n)}(t, u)\right\}_{n}$ admits, as $n$ goes to infinity, a nonnegative limit $f(t, \cdot) \in L^{1}\left(D_{u}, d u\right)$ such that $\mathbb{E}_{0}[f](t)=1$.

Proof. Since $f_{0}$ is a nonnegative function, then $f^{(n)}(t, u)>0$, $\forall n \geq 1$. Moreover,

$$
f^{(2)}(t, u)=e^{-\Lambda(t)} f_{0}\left(\varphi_{t}^{-1}(u)\right) \geq 0=f^{(1)}(t, u),
$$

and $\mathbb{E}_{0}\left[f^{(2)}\right](t)=e^{-\eta t} \leq 1$.

We now prove by induction that the sequence $\left\{f^{(n)}(t, \cdot)\right\}_{n}$ is monotone, and specifically

$$
f^{(n)}(t, u) \geq f^{(n-1)}(t, u), \quad \forall u \in D_{u}, \forall n \geq 1,
$$

and $\mathbb{E}_{0}[f](t) \leq 1$.

Assume as the induction hypothesis that, for some $n \geq 3$, we have $f^{(n-1)} \geq f^{(n-2)}$ and $\mathbb{E}_{0}\left[f^{(n-1)}\right](t) \leq 1$. Then,

$$
\begin{aligned}
& f^{(n)}-f^{(n-1)} \\
& =e^{-\Lambda(t)} \int_{0}^{t} e^{\Lambda(\alpha)}\left(G\left[f^{(n-1)}, f_{\tau}^{(n-1)}\right]-G\left[f^{(n-2)}, f_{\tau}^{(n-2)}\right]\right) \\
& \quad \times\left(\alpha, \varphi_{\alpha} \circ \varphi_{t}^{-1}(u)\right) d \alpha .
\end{aligned}
$$

Taking into account property (8), equation (36) thus reads as follows:

$$
\begin{aligned}
e^{-\Lambda(t)} \int_{0}^{t} e^{\Lambda(\alpha)}( & {\left[f^{(n-1)}-f^{(n-2)}, f_{\tau}^{(n-1)}\right] } \\
+ & \left.G\left[f^{(n-2)}, f_{\tau}^{(n-1)}-f_{\tau}^{(n-2)}\right]\right) d \alpha,
\end{aligned}
$$


and by using the induction hypothesis we conclude that the sequence $\left\{f^{(n)}(t, \cdot)\right\}_{n}$ is monotone. Moreover,

$$
\begin{aligned}
& \int_{D_{u}} f^{(n)}(t, u) d u \\
& =e^{-\eta t}+e^{-\eta t} \int_{0}^{t} e^{\eta \alpha} \int_{D_{u}} G\left[f^{(n-1)}, f_{\tau}^{(n-1)}\right](\alpha, u) d \alpha d u .
\end{aligned}
$$

Taking into account property (12), we have

$$
\begin{aligned}
& \mathbb{E}_{0}\left[f^{(n)}\right](t) \\
& =e^{-\eta t}+\eta e^{-\eta t} \int_{0}^{t} e^{\eta \alpha} \mathbb{E}_{0}\left[f^{(n-1)}\right](\alpha) \\
& \quad \times \mathbb{E}_{0}\left[f^{(n-1)}\right](\alpha-\tau) d \alpha,
\end{aligned}
$$

and by using the induction hypothesis we have

$$
\mathbb{E}_{0}\left[f^{(n)}\right](t) \leq e^{-\eta t}+\eta e^{-\eta t} \int_{0}^{t} e^{\eta \alpha} d \alpha=1 .
$$

Bearing all of the above in mind, we conclude that the sequence $\left\{f^{(n)}(t, \cdot)\right\}_{n}$ has a nonnegative limit $f(t, \cdot) \in L^{1}\left(D_{u}\right)$ such that $f^{(n)} \rightarrow f$, as $n \rightarrow \infty$. Then, the Levi theorem implies that $f$ satisfies the following equation:

$$
\begin{aligned}
& \mathbb{E}_{0}[f](t) \\
& \quad=e^{-\eta t}+\eta e^{-\eta t} \int_{0}^{t} e^{\eta \alpha} \mathbb{E}_{0}[f](\alpha) \mathbb{E}_{0}[f](\alpha-\tau) d \alpha
\end{aligned}
$$

whose unique solution is $\mathbb{E}_{0}[f](t)=1$. Therefore, the lemma is completely proved.

The main result of this paper is the following.

Theorem 8. Let $f_{0}$ be a given nonnegative function such that $\mathbb{E}_{0}\left[f_{0}\right]=1$. Then, there exists a unique nonnegative mild solution

$$
f \in C\left((0, \infty) ; L^{1}\left(D_{u}, d u\right)\right)
$$

to the Cauchy problem (1).

Proof. Since Lemma 7 states that $f$ solves (31), in order to prove that $f$ is a mild solution of (1), it is enough to show that $\mathbb{E}_{1}[f](t)=\beta(t)$. In order to prove that $\mathbb{E}_{1}[f](t)=\beta(t)$, we consider the following successive approximations sequence:

$$
\begin{gathered}
g^{(1)}(t, u)=f_{0}(u), \\
g^{(n)}(t, u)=\Phi_{f_{0}}\left[g^{(n-1)}, g_{\tau}^{(n-1)}\right](t, u), \quad n>1,
\end{gathered}
$$

or the following equivalent form of (43):

$$
\begin{aligned}
e^{\Lambda(t)} & g^{(n)}\left(t, \varphi_{t}(u)\right) \\
& =f_{0}(u)+\int_{0}^{t} e^{\Lambda(\tau)} G\left[g^{(n-1)}, g_{\tau}^{(n-1)}\right]\left(\alpha, \varphi_{\alpha}(u)\right) d \alpha .
\end{aligned}
$$

The assumption on $f_{0}$ implies that the zero-order moment $\mathbb{E}_{0}\left[g^{(1)}\right](t)=1$. Assume now as induction hypothesis that $\mathbb{E}_{0}\left[g^{(n-1)}\right](t)=1$, for some $n \geq 2$. Integrating both sides of (44) over $D_{u}$ with respect to $u$, and using (28), we obtain

$$
\begin{aligned}
e^{\eta t} \int_{D_{u}} & g^{(n)}(t, u) d u \\
= & \int_{D_{u}} f_{0}(u) d u \\
& \quad+\int_{0}^{t} e^{\eta \alpha} \int_{D_{u}} G\left[g^{(n-1)}, g_{\tau}^{(n-1)}\right](\alpha, u) d \alpha d u .
\end{aligned}
$$

Taking into account property (12) and by using the induction hypothesis, the right-hand side of (45) thus reads as follows:

$$
\begin{aligned}
& e^{\eta t} \int_{D_{u}} g^{(n)}(t, u) d u \\
& \quad=\int_{D_{u}} f_{0}(u) d u+\eta \int_{0}^{t} e^{\eta \alpha} d \alpha=e^{\eta t} .
\end{aligned}
$$

Therefore, for all $t \geq 0$, we have $\mathbb{E}_{0}\left[g^{(n)}\right](t)=1$.

Multiplying both sides of (44) by $u$, and integrating over $D_{u}$ with respect to $u$, we have

$$
\begin{gathered}
\int_{D_{u}} u g^{(n)}(t, u) d u \\
=e^{-\Lambda(t)} \int_{D_{u}} \int_{0}^{t} e^{\Lambda(\alpha)} u G\left[g^{(n-1)}, g_{\tau}^{(n-1)}\right] \\
\quad \times\left(\alpha, \varphi_{\alpha} \circ \varphi_{t}^{-1}(u)\right) d \alpha d u \\
+e^{-\Lambda(t)} \int_{D_{u}} u f_{0}\left(\varphi_{t}^{-1}(u)\right) d u .
\end{gathered}
$$

Taking into account (12), (13) and repeating the computations developed in [6], it is easy to prove by induction on $n$ that

$$
\mathbb{E}_{1}\left[g^{(n)}\right](t)=\int_{D_{u}} u g^{(n)}(t, u) d u=\beta(t)
$$

Multiplying both sides of (44) by $u^{2}$ and integrating over $D_{u}$ with respect to $u$, we have

$$
\begin{aligned}
e^{\Lambda(t)} & \int_{D_{u}} u^{2} g^{(n)}\left(t, \varphi_{t}(u)\right) d u \\
= & \int_{D_{u}} \int_{0}^{t} e^{\Lambda(\alpha)} u^{2} G\left[g^{(n-1)}, g_{\tau}^{(n-1)}\right]\left(\alpha, \varphi_{\alpha}(u)\right) d \alpha d u \\
& +\int_{D_{u}} u^{2} f_{0}(u) d u,
\end{aligned}
$$

and according to [6] it is easy to prove by induction on $n$ that

$$
\mathbb{E}_{2}\left[g^{(n)}\right](t)=\int_{D_{u}} u^{2} g^{(n)}(t, u) d u=1
$$


Let $\sum=\left\{u \in D_{u}: f(t, u) \geq g^{(n)}(t, u)\right\}$. Then,

$$
\begin{aligned}
\int_{D_{u}}\left|f-g^{(n)}\right| d u & \\
& =2 \int_{\Sigma}\left(f-g^{(n)}\right) d u-\int_{D_{u}}\left(f-g^{(n)}\right) d u .
\end{aligned}
$$

Since $\mathbb{E}_{0}[f](t)=\mathbb{E}_{0}\left[g^{(n)}\right](t)=1$ and by construction $f^{n} \leq$ $g^{n}$, we have

$$
\begin{aligned}
\int_{D_{u}}\left|f-g^{(n)}\right| d u & \\
=2 \int_{\Sigma}\left(f-g^{(n)}\right) d u \leq 2 \int_{D_{u}}\left(f-f^{(n)}\right) d u & \longrightarrow 0 \\
n \longrightarrow \infty &
\end{aligned}
$$

Therefore, $g^{(n)} \rightarrow f$ in $L^{1}\left(D_{u}, d u\right)$, and since $\mathbb{E}_{2}\left[g^{(n)}\right](t)$ is bounded, then $\mathbb{E}_{1}[f](t)=\beta(t)$.

We now prove the uniqueness of the solution. Let $\bar{f}(t, u)$ be any solution to (31). The positivity of the operators $G$ and $\Phi_{f_{0}}$ implies that, for all $n, f^{(n)}(t, u) \leq \bar{f}(t, u)$, and then $f(t, u) \leq \bar{f}(t, u)$. Since $\mathbb{E}_{0}[f](t)=\mathbb{E}_{0}[\bar{f}](t)=1$, we thus have $\bar{f}=f$.

Corollary 9. Let $f_{0}$ be a given nonnegative function such that

(i) $\mathbb{E}_{0}\left[f_{0}\right]=1$;

(ii) $\int_{D_{u}}|u|^{3} f_{0}(u) d u<\infty$.

Then, the mild solution $f$ of the Cauchy problem (1) belongs to $\mathscr{M}\left(D_{u}\right)$ where

$$
\begin{aligned}
& \mathscr{M}\left(D_{u}\right)=\left\{f=f(t, u):[0, \infty) \times D_{u} \longrightarrow \mathbb{R}^{+}\right. \\
&\text {such that } \left.\mathbb{E}_{0}[f](t)=\mathbb{E}_{2}[f](t)=1\right\} .
\end{aligned}
$$

Proof. It is sufficient to remember (24) and note that assumption (ii) implies that

$$
\int_{D_{u}}|u|^{3} g^{(n)}(t, u) d u<\infty
$$

which allows us to conclude that $E_{2}[f](t)=1$.

\section{Applications and Research Perspectives}

The main goal of the present paper refers to the proof of the global existence of mild solutions of a thermostatted partial integrodifferential equation with time delay. As already mentioned in Section 2, this equation can be proposed for the modeling of complex systems in nature and society where only the interactions at the microscopic scales are affected by time delay. Specifically, the partial integrodifferential equation with time delay

$$
\begin{aligned}
\partial_{t} f(t, u) & =F \partial_{u}\left(f(t, u)\left(1-u \int_{D_{u}} u f(t, u) d u\right)\right) \\
& =\eta J\left[f, f_{r}\right](t, u)
\end{aligned}
$$

can be proposed as a general mathematical framework for the modeling of complex systems composed by a large number of interacting particles and subjected to the external force field $F$. The overall state of the system is described by the distribution function $f$ (statistical description). Particles are able to express a specific function; this ability of the particles is modeled by the variable $u \in D_{u} \subseteq \mathbb{R}$. Moreover, $\eta$ is the encounter rate between particles with states $u_{*}$ (or $u$ ) and $u^{*}$. Finally, $\mathscr{A}$ is the probability density that a particle with state $u_{*}$ ends up into the state $u$ after the interaction with the particle with state $u^{*}$. The action of the external force field is controlled by the thermostat term that, as shown in Section 4, maintains constant the first-order and the secondorder moments (number density and activation energy of the system).

From the applications viewpoint, we consider a simple model for the evolution of malignancy in tumor cells. Specifically, we assume that the variable $u$ models the magnitude of the malignancy of tumor cells and $\mathscr{A}$ is a delta Dirac function (deterministic output $m\left(u_{*}, u^{*}\right)$ of a pair interaction) depending on the microscopic state of the interacting particles:

$$
\mathscr{A}\left(u_{*}, u^{*}, u\right)=\delta\left(u-m\left(u_{*}, u^{*}\right)\right)
$$

and finally we assume that the malignancy of tumor cells increases when cells interact with each other with rate $\eta$. Accordingly, we have

$$
m\left(u_{*}, u^{*}\right)=u_{*}+\epsilon,
$$

where $\epsilon$ is a positive parameter. Bearing all of the above in mind, the integral operator $J\left[f, f_{\tau}\right]$ reads as follows:

$$
J\left[f, f_{\tau}\right]=[f(t, u-\epsilon)-f(t, u)] \int_{D_{u}} f(t-\tau, u) d u,
$$

and then delayed equation (55) now reads as follows:

$$
\begin{array}{r}
\partial_{t} f(t, u)+F \partial_{u}\left(f(t, u)\left(1-u \int_{D_{u}} u f(t, u) d u\right)\right) \\
=\eta[f(t, u-\epsilon)-f(t, u)] \int_{D_{u}} f(t-\tau, u) d u .
\end{array}
$$

However, thermostatted equation (55) does not include the role of the space and velocity variables; then, applications refer to the modeling of complex phenomena that are homogeneous in space and velocity. The mathematical analysis performed in the present paper has to be thus generalized for taking also into account the dynamics described by these variables. Moreover, (55) refers to complex systems where the mutual interactions do not produce modification in the number density (conservative interactions).

Research perspectives include the possibility of performing an asymptotic analysis by parabolic (low-field) and hyperbolic (high-field) scalings (see [24-34]) with the aim of obtaining the dynamics of the system at the macroscopic scale. This is a work in progress and results will be presented in due course. 


\section{Conflict of Interests}

The authors declare that there is no conflict of interests regarding the publication of this paper.

\section{Acknowledgment}

Carlo Bianca and Annie Lemarchand were partially supported by L'Agence Nationale de la Recherche (ANR T-KiNeT Project).

\section{References}

[1] Y. Bar-Yam, Dynamics of Complex Systems, Studies in Nonlinearity, Westview Press, 2003.

[2] C. Bianca, "Thermostatted kinetic equations as models for complex systems in physics and life sciences," Physics of Life Reviews, vol. 9, no. 4, pp. 359-399, 2012.

[3] C. Bianca, "How do mutative events modify moments evolution in thermostatted kinetic models?" Communications in Nonlinear Science and Numerical Simulation, vol. 19, no. 7, pp. 21552159, 2014.

[4] C. Bianca, C. Dogbe, and L. Guerrini, "A thermostatted kinetic framework with particle refuge for the modeling of tumors hiding," Applied Mathematics \& Information, vol. 8, no. 2, pp. 469-473, 2014.

[5] C. Bianca, M. Ferrara, and L. Guerrini, "High-order moments conservation in thermostatted kinetic models," Journal of Global Optimization, vol. 58, no. 2, pp. 389-404, 2014.

[6] C. Bianca, "Modeling complex systems by functional subsystems representation and thermostatted-KTAP methods," Applied Mathematics and Information Sciences, vol. 6, no. 3, pp. 495-499, 2012.

[7] P. J. Cunningham and W. J. Wangersky, Time Lag in Population Models, Yale, 1958.

[8] T. Erneux, Applied Delay Differential Equations, Springer, New York, NY, USA, 2009.

[9] C. T. H. Baker, G. A. Bocharov, and C. A. H. Paul, "Mathematical modelling of the interleukin-2 T-cell system: a comparative study of approaches based on ordinary and delay differential equations," Journal of Theoretical Medicine, vol. 2, pp. 117-128, 1997.

[10] J. Bélair, M. C. Mackey, and J. M. Mahay, "Age-structured and two-delay models for erythropoiesis," Mathematical Biosciences, vol. 128, no. 1-2, pp. 317-346, 1995.

[11] Y. Kuang, Delay Differential Equations with Applications in Popula tion Dynamics, Mathematics in Science and Engineering, Academic Press, Boston, Mass, USA, 1993.

[12] H. M. Byrne, "The effect of time delays on the dynamics of avascular tumor growth," Mathematical Biosciences, vol. 144, no. 2, pp. 83-117, 1997.

[13] M. J. Piotrowska, "Hopf bifurcation in a solid avascular tumour growth model with two discrete delays," Mathematical and Computer Modelling, vol. 47, no. 5-6, pp. 597-603, 2008.

[14] C. Bianca, M. Ferrara, and L. Guerrini, "Hopf bifurcations in a delayed-energy-based model of capital accumulation," Applied Mathematics and Information Sciences, vol. 7, no. 1, pp. 139-143, 2013.

[15] C. Bianca and L. Guerrini, "Existence of limit cycles in the Solow model with delayed-logistic population growth," The Scientific World Journal, vol. 2014, Article ID 207806, 8 pages, 2014.

[16] C. Bianca, M. Ferrara, and L. Guerrini, "Qualitative analysis of a retarded mathematical framework with applications to living systems," Abstract and Applied Analysis, vol. 2013, Article ID 736058, 7 pages, 2013.

[17] Y. Ma, "Global Hopf bifurcation in the Leslie-Gower predatorprey model with two delays," Nonlinear Analysis: Real World Applications, vol. 13, no. 1, pp. 370-375, 2012.

[18] Y. Song, J. Wei, and M. Han, "Local and global Hopf bifurcation in a delayed hematopoiesis model," International Journal of Bifurcation and Chaos in Applied Sciences and Engineering, vol. 14, no. 11, pp. 3909-3919, 2004.

[19] J. Wei, "Bifurcation analysis in a scalar delay differential equation," Nonlinearity, vol. 20, no. 11, pp. 2483-2498, 2007.

[20] T. Chen, X. Li, and J. He, "Complex dynamics of credit risk contagion with time-delay and correlated noises," Abstract and Applied Analysis, vol. 2014, Article ID 456764, 10 pages, 2014.

[21] K. F. Gauss, "Uber ein Neues Allgemeines Grundgesatz der Mechanik (On a New Fundamental Law of Mechanics)," Journal für die Reine und Angewandte Mathematik, vol. 4, pp. 232-235, 1829.

[22] G. P. Morriss and C. P. Dettmann, "Thermostats: analysis and application," Chaos, vol. 8, no. 2, pp. 321-336, 1998.

[23] D. Ruelle, "Smooth dynamics and new theoretical ideas in nonequilibrium statistical mechanics," Journal of Statistical Physics, vol. 95, no. 1-2, pp. 393-468, 1999.

[24] C. Bardos, F. Golse, and D. Levermore, "Fluid dynamic limits of kinetic equations. I. Formal derivations," Journal of Statistical Physics, vol. 63, no. 1-2, pp. 323-344, 1991.

[25] P. Lions and N. Masmoudi, "From the Boltzmann equations to the equations of incompressible fluid mechanics-I," Archive for Rational Mechanics and Analysis, vol. 158, no. 3, pp. 173-193, 2001.

[26] C. Dogbe, "Fluid dynamic limits for gas mixture. I. Formal derivations," Mathematical Models and Methods in Applied Sciences, vol. 18, no. 9, pp. 1633-1672, 2008.

[27] T. Hillen and H. G. Othmer, "The diffusion limit of transport equations derived from velocity-jump processes," SIAM Journal on Applied Mathematics, vol. 61, no. 3, pp. 751-775, 2000.

[28] J. Soler, L. L. Bonilla, O. Sánchez, and T. Goudon, "Low-field limit for a nonlinear discrete drift-diffusion model arising in semiconductor superlattices theory," SIAM Journal on Applied Mathematics, vol. 64, no. 5, pp. 1526-1549, 2004.

[29] F. Poupaud, "Runaway phenomena and fluid approximation under high fields in semiconductor kinetic theory," Zeitschrift für Angewandte Mathematik und Mechanik, vol. 72, no. 8, pp. 359-372, 1992.

[30] P. Degond and B. Wennberg, "Mass and energy balance laws derived from high-field limits of thermostatted Boltzmann equations," Communications in Mathematical Sciences, vol. 5, no. 2, pp. 355-382, 2007.

[31] H. Grad, "Asymptotic theory of the Boltzmann equation," in Rarefied Gas Dynamics (Proceedings of the 3rd International Symposium), vol. 1, pp. 26-59, Palais de l'UNESCO, Paris, France, 1962.

[32] C. Bianca and A. Lemarchand, "Density evolution by the lowfield limit of kinetic frameworks with thermostat and mutations," Communications in Nonlinear Science and Numerical Simulation, vol. 20, no. 1, pp. 14-23, 2015.

[33] C. Cercignani, The Boltzmann Equation and Its Applications, vol. 67 of Applied Mathematical Sciences, Springer, New York, NY. USA, 1988.

[34] G. C. Papanicolaou, "Asymptotic analysis of transport processes," Bulletin of the American Mathematical Society, vol. 81, pp. 330-392, 1975. 


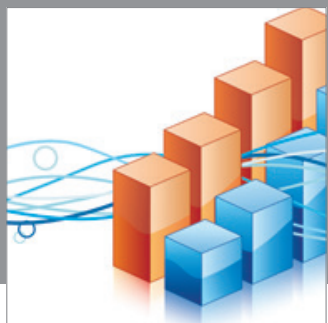

Advances in

Operations Research

mansans

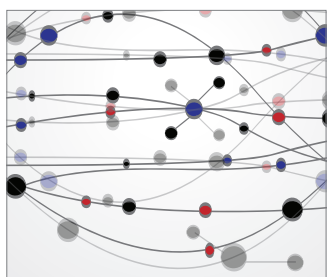

The Scientific World Journal
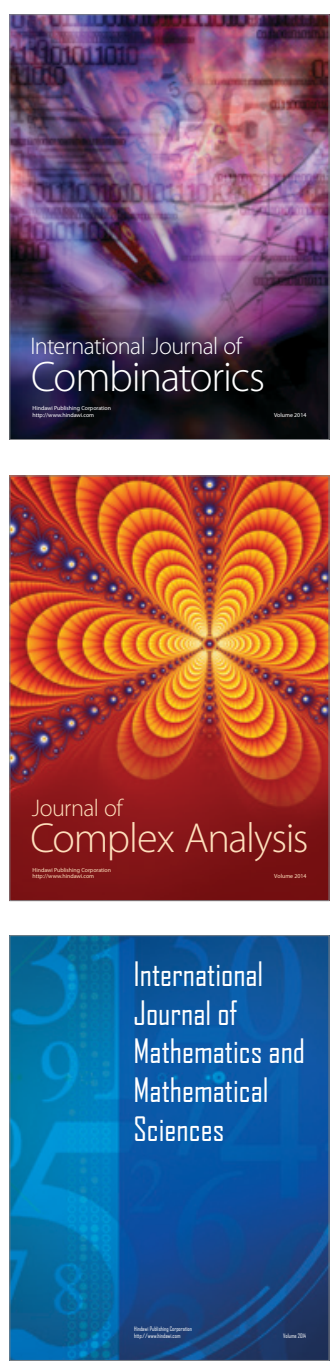
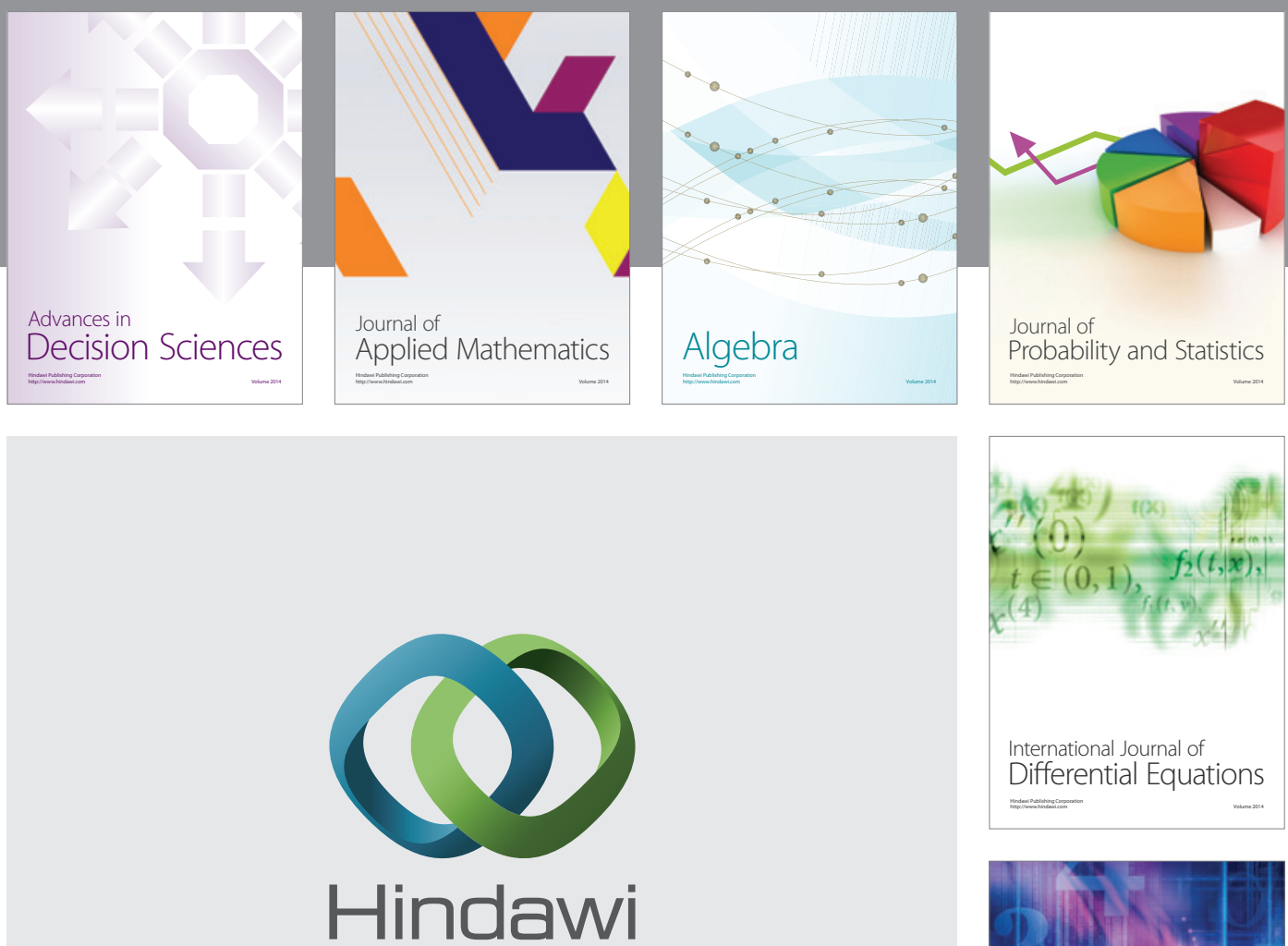

Submit your manuscripts at http://www.hindawi.com


Journal of

Function Spaces



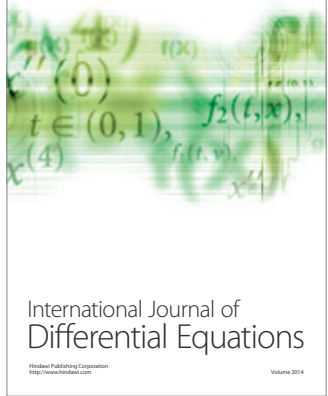
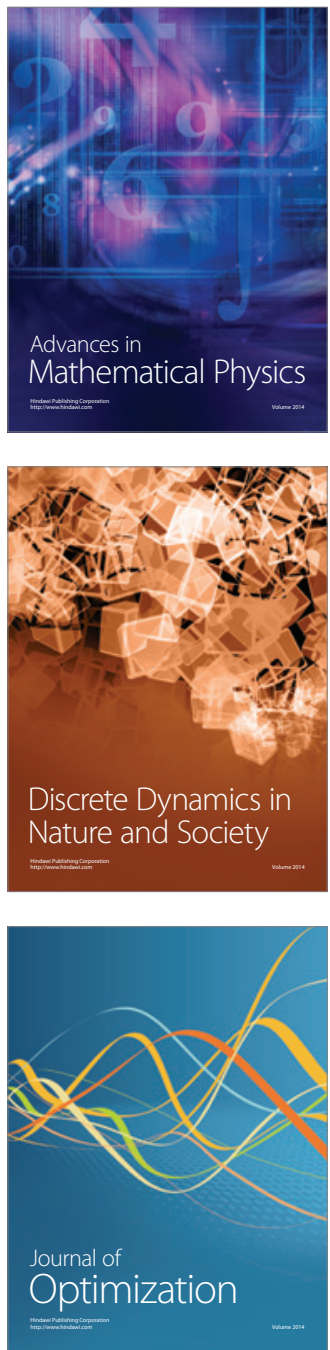